\title{
Tannic Acid-Based Multifunctional Hydrogels with Facile Adjustable Adhesion and Cohesion Contributed by Polyphenol Supramolecular Chemistry
}

\author{
Hailong Fan, Jiahui Wang, Qiuya Zhang, and Zhaoxia Jin*(i) \\ Department of Chemistry, Renmin University of China, No. 59 Zhongguancun Street, Haidian District, Beijing 100872, P. R. China
}

Supporting Information

ABSTRACT: Adhesiveness of hydrogels depends on the balance and synergy of their cohesion and adhesion. However, it is a challenge to fabricate catechol-based hydrogels with high adhesiveness because the required condition for cohesion and adhesion of these hydrogels is in conflict with each other: strong cohesion (gelation) requires a weak basic condition, whereas strong adhesion requires an acidic condition. Here, we demonstrated that by utilizing polyphenol supramolecular chemistry, the coexistence of strong cohesion and adhesion can be achieved in a hydrogel via the one-pot method. Poly(dimethyl diallyl ammonium chloride)/tannic acid (PDDA/TA) hydrogel has been studied as a proof of concept.
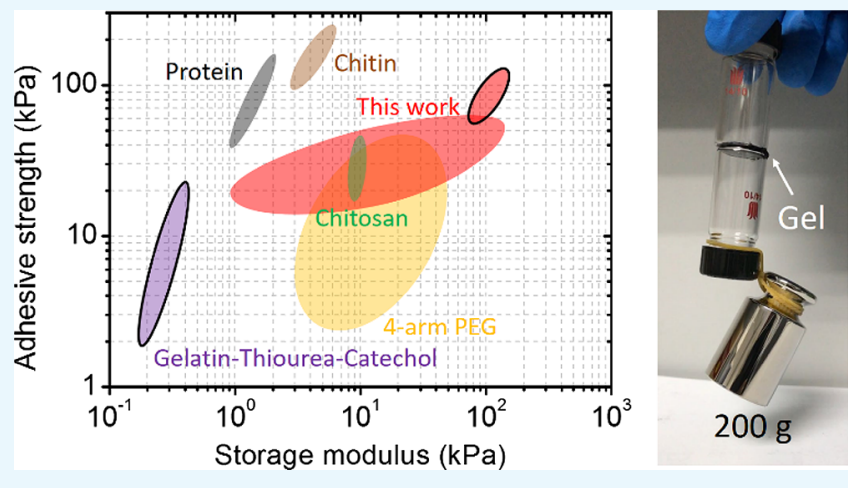
Compared with catechol moieties that covalently grafted on polymer chains, TA can bring high density of pyrogallol/catechol functional groups for polymers via a noncovalent pathway, as well as high acidity in the system. As a result, the cohesion of the hydrogel is enhanced significantly, the highest storage moduli can reach up to ca. $0.15 \mathrm{MPa}$; besides, the high acidity of the hydrogel prevents pyrogallol/catechol groups from oxidation and guarantees strong adhesion; thus, the hydrogel can adhere to diverse substrates steadily, including tissues, glass, metals, and plastic. Moreover, because of the adjustable adhesiveness via changing the $\mathrm{pH}$, the PDDA/TA hydrogel becomes a unique system with patternable adhesiveness. In addition, the hydrogel has rapid self-healing and high ionic conductivity $\left(\sim 4.3 \mathrm{~S} \mathrm{~m}^{-1}\right)$. This study demonstrates that utilizing polyphenol chemistry in the construction of hydrogels opens a new path toward multifunctional hydrogels with improved properties.

\section{INTRODUCTION}

Mussel is well-known for its ability to cling to diverse surfaces. Catecholic amino acid 3,4-dihydroxy-L-phenylalanine (DOPA) is the key component of its adhesive foot proteins ( $\mathrm{mfps}){ }^{1-3}$ The catechol chemistry contributes to the universal adhesion as a synergic system: first, the catechol group of $\mathrm{mfp}-3$ and $\mathrm{mfp}-5$ can bond to substrates through the formation of reversible noncovalent or irreversible covalent interactions, leading to high adhesion of mfps; ${ }^{1}$ second, the catechol-rich mfp-1 and $\mathrm{mfp}-2$ form bis and tris complexes with metal ions, resulting in strong cohesion of mfps. ${ }^{4,5}$ To overcome the weakening adhesiveness brought by the catechol oxidation, mussels generate a seawater-free, acidified, and isolated environment to ensure strong adhesion of $\mathrm{mfp}-3$ and $\mathrm{mfp}-5$ and further suppress the adverse effects of catechol oxidation by adopting thiol-rich mfp- 6 as a reducing agent. ${ }^{6-8}$ By forming a fine and hierarchical structure to balance adhesion and cohesion, $\mathrm{mfps}$ help mussels to adhere to the rock steadily.

To mimic adhesive functionality of the DOPA-rich mfps, different polymers containing catechol groups have been synthesized, which can be further cross-linked into hydrogels with good wet adhesiveness. $^{9-12}$ In general, the formation of catechol-based hydrogels requires elevating the $\mathrm{pH}$ of polymer solutions from acidic to basic condition, in which catechol groups work as the cross-link point through either polymerization or multicoordinate bonds with metal ions. ${ }^{11}$ However, compared with sophisticated engineering of mussel byssus, the man-made catechol-based hydrogel is too simple in both chemical composition and hierarchical structures to have strong cohesion and adhesion simultaneously, resulting in low mechanical strength and weak adhesiveness. In particular, although catechol-based hydrogels exhibit good adhesiveness to tissues by forming covalent bonds, they present poor adhesiveness to engineered solids. ${ }^{3}$ This is because in catechol-based hydrogels, catechol groups are either occupied by metal ions or oxidized into quinone or both; as a consequence, the drastic reduction of the hydrogen bond of catechol weakens the adhesiveness of the hydrogel to engineered solid surfaces (Figure 1a). ${ }^{13-15}$ Besides, it is difficult to synthesize polymers bearing a high amount of associate groups because of the technical limit. ${ }^{16,17}$ To overcome the above-mentioned limitation of catechol-based hydrogels, we

Received: July 25, 2017

Accepted: September 5, 2017

Published: October 12, 2017 
(a) Catechol-based hydrogels

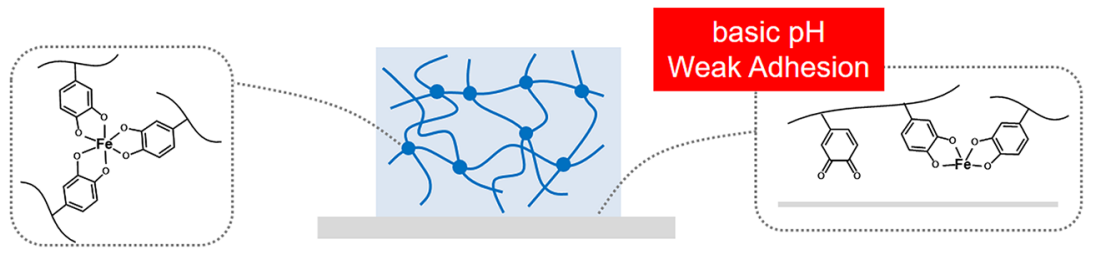

(b) Tannic acid-based hydrogels

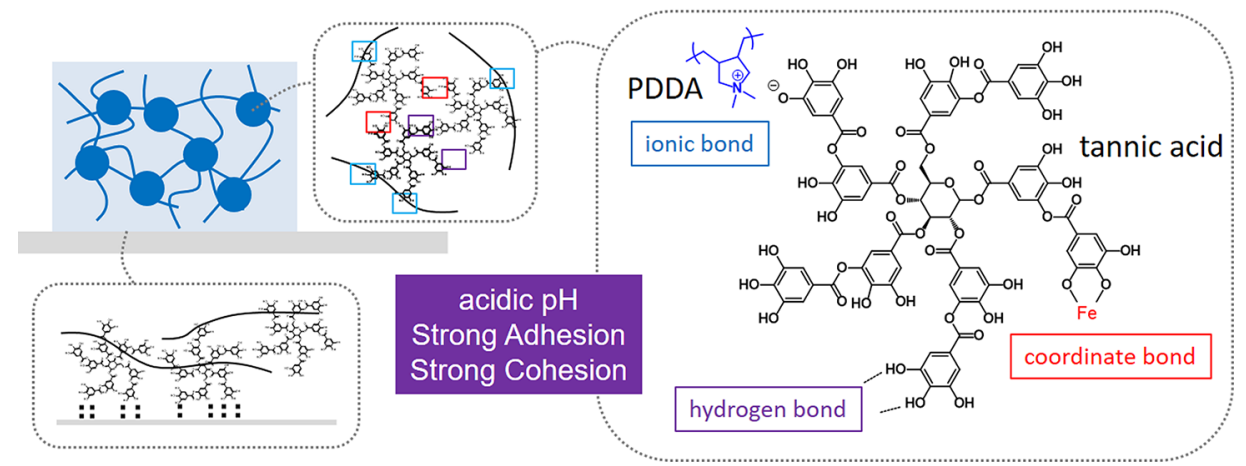

Figure 1. Schematic comparison of the molecular-level mechanism of catechol-based hydrogels and TA-based hydrogels on adhesion and cohesion. (a) Schematic illustration of man-made catechol-based hydrogels. In catechol-based hydrogels, the tris complex of catechol and metal ion works as a cross-linker in hydrogels. However, the basic $\mathrm{pH}$ environment reduces the adhesion of hydrogels. (b) Schematic illustration of the TA-based supramolecular hydrogel presented in this study. The high density of pyrogallol/catechol groups and the dendritic structure of TA provide strong adhesion and cohesion in the hydrogel.
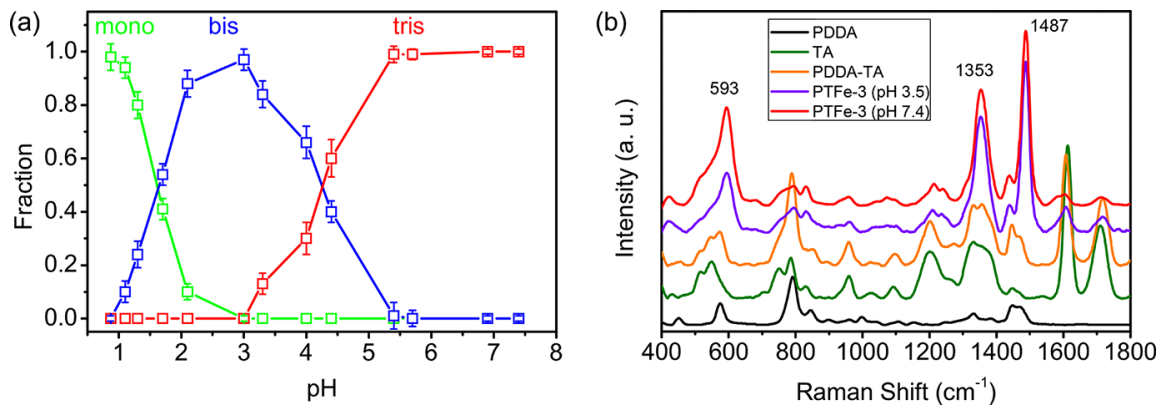

Figure 2. (a) Relative fractions of mono- (green), bis- (blue), and tris-catechol/ $\mathrm{Fe}^{3+}$ (red) complexes in the PTFe hydrogel as a function of the $\mathrm{pH}$. (b) Raman spectra of PDDA, TA, PDDA/TA mixture, and PTFe-3 hydrogels with different $\mathrm{pH}$ values.

propose a new strategy based on polyphenol for developing robust and adhesive hydrogels.

Tannic acid (TA) is a natural polyphenol that can be extracted from various plants. It has valuable properties such as antioxidant, antibacterial, and biodegradability based on the viewpoint of functional materials. ${ }^{18,19}$ TA contains five pyrogallol and five catechol groups, which can provide multiple bonding sites with diverse interactions, including hydrogen bond, ionic bond, coordinate bond, and hydrophobic interactions. $^{20-22}$ The formation of metal-phenolic networks (MPNs) via the coordination between catechol/galloyl functional groups and metal ions has been explored by Caruso et al. $^{23-27}$ The assembly of MPN has been applied in the generation of the thin film or particles with tailored properties ${ }^{23}$ and in the formation of novel metallogels. ${ }^{26}$ Extending the application of various bonding modes of TA in materials science has been booming recently.

In our previous study, we have demonstrated that TA molecules can be functionalized on polymer chains via a noncovalent pathway, and they behave like covalently connected catechol groups in cross-linking polymers to hydrogels. $^{22}$ Herein, we first report a multifunctional hydrogel with adjustable mechanical strength and adhesiveness generated via adopting polyphenol chemistry. TA-based polyphenol hydrogels provide solutions aiming at the weakness of catechol-based hydrogels, as illustrated in Figure $1 \mathrm{~b}$. In this system, TA was used as a multiple pyrogallol-based dynamic associate moiety, which interacts with poly(dimethyl diallyl ammonium chloride) (PDDA) chains through ionic bonds on the one hand and cross-links the polymer chains into networks by $\mathrm{Fe}^{\mathrm{III}}$ via coordination bonds on the other. Variable cross-link density can be achieved by tuning the TA/PDDA weight ratios through supramolecular assembly. As consequences: (i) the cohesion of hydrogels is dramatically enhanced, and the highest storage modulus achieves ca. $0.15 \mathrm{MPa}$; (ii) PDDA/TA/ $\mathrm{Fe}^{\mathrm{III}}(\mathrm{PTFe})$ gel can be formed in a strong acidic $\mathrm{pH}$ range $(\mathrm{pH}$ 1-3), which prevents pyrogallol/catechol groups from oxidation, so that the hydrogels possess strong adhesiveness. 

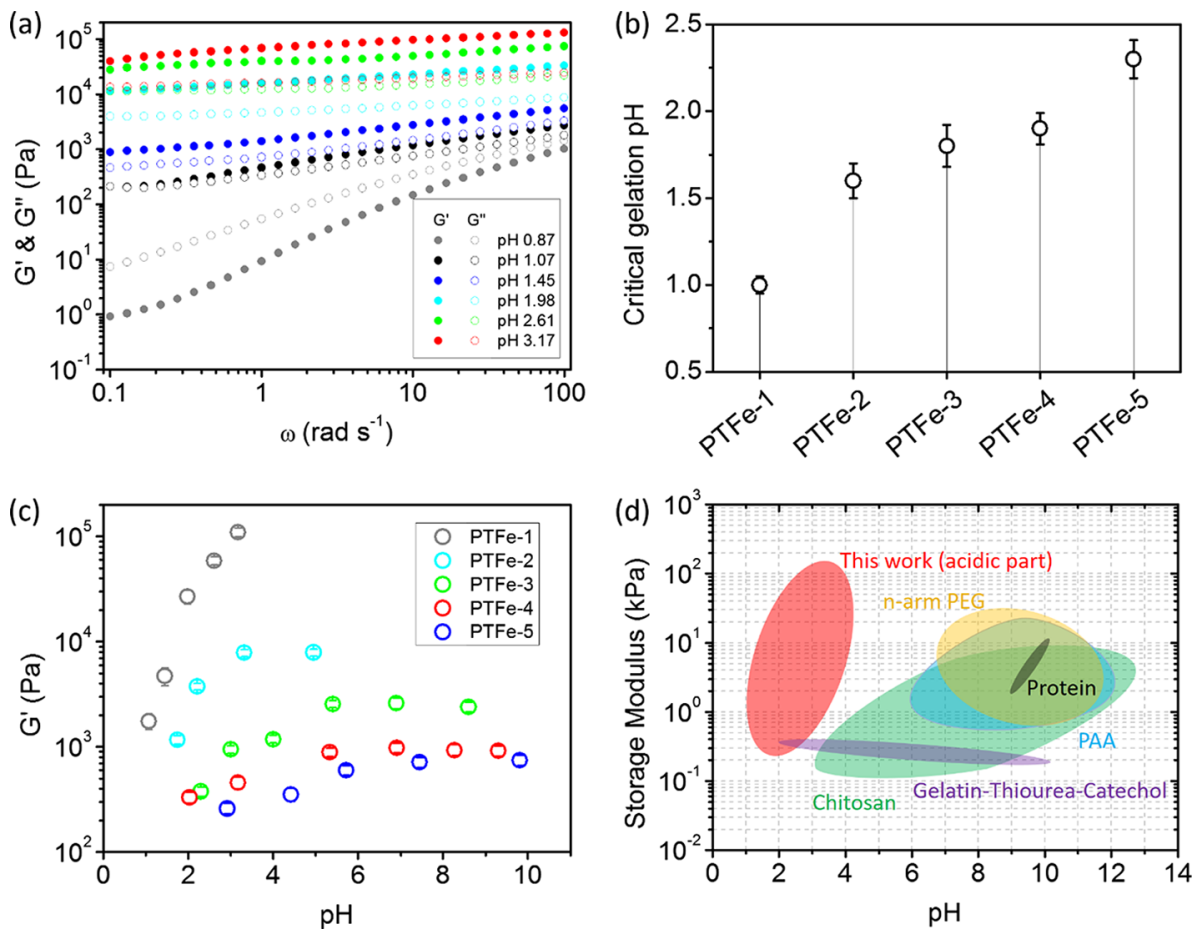

Figure 3. (a) The storage $G^{\prime}$ and loss $G^{\prime \prime}$ moduli of PTFe-1 hydrogels with angular frequency sweeps between 0.1 and 100 rad s $\mathrm{s}^{-1}$. (b) Critical gelation $\mathrm{pH}$ of PTFe hydrogels. (c) Storage modulus $\left(G^{\prime}\right)$ of PTFe hydrogels plotted as a function of $\mathrm{pH}$ at an angular frequency of 25 rad s ${ }^{-1}$ and a strain of 1\%. (d) Storage modulus chart for various catechol-based hydrogels in different $\mathrm{pH}$ values. Hydrogels include the gel prepared in this work marked as red (acidic part), catechol-based chitosan hydrogels as green, ${ }^{29,30}$ gelatin/thiourea/catechol hydrogels as purple, ${ }^{31}$ catechol-based 4- or 8arm polyethylene glycol (PEG) hydrogels as yellow, ${ }^{9,32,33}$ catechol-based protein or peptide hydrogels as dark gray, ${ }^{34,35}$ and catechol-based polyallylamine (PAA) hydrogels as blue. ${ }^{36}$

The hydrogel exhibited very strong adhesive strength to diverse substrates, including tissues, glass, metals, and plastic. In addition, this PDDA/TA/Fe ${ }^{\text {III }}$ hydrogel has rapid self-healing and high ionic conductivity $\left(\sim 4.3 \mathrm{~S} \mathrm{~m}^{-1}\right)$. Because polyphenol is a kind of a nature product, further investigation of polyphenol chemistry in materials science will promote the fabrication of green materials with versatile functionalities.

\section{RESULTS AND DISCUSSION}

2.1. Formation of PDDA/TA/Fe (PTFe) Hydrogels. The color of the catechol/ $\mathrm{Fe}^{3+}$ complex depends on the coordination number of $\mathrm{Fe}^{3+}$ to catechol, which is influenced by its $\mathrm{pH}$ value. ${ }^{9} \mathrm{UV}-$ vis spectroscopy was used to monitor the catechol $/ \mathrm{Fe}^{3+}$ stoichiometry of these PDDA/TA/Fe ${ }^{\mathrm{III}}$ mixtures (Figure S1). Figure 2a shows the relative fractions of three coordination states of catechol $/ \mathrm{Fe}^{3+}$, which were calculated based on the absorbance data in UV-vis spectra of mixtures. When the $\mathrm{pH}$ was below 1.3, the mixture was green because of the dominant monocatechol iron complexes. When the $\mathrm{pH}$ increased above 1.7, the mixture turned blue/purple because of the formation of bis complexes. When the $\mathrm{pH}$ further increased above 5.4, the mixture turned red after the formation of tris complexes. Compared with the single catechol group, the bis and tris complexes of TA/ $\mathrm{Fe}^{3+}$ are formed at lower $\mathrm{pH}$ values, which is caused by the lower $\mathrm{p} K_{\mathrm{a}}$ value of TA $\left(\mathrm{p} K_{\mathrm{a}} 8.5\right){ }^{11,21}$ Figure $2 \mathrm{~b}$ presents the Raman characteristic of $\mathrm{Fe}^{3+} /$ catechol coordination. Compared with those of PDDA, TA, and PDDA/ TA mixture, the PTFe-3gel shows significant differences in its spectrum. The peaks around 1353 and $1487 \mathrm{~cm}^{-1}$ belong to $\mathrm{Fe}^{3+} /$ catechol complex ring vibrations, and the peak around $593 \mathrm{~cm}^{-1}$ is assigned specifically to the bidentate chelation of
$\mathrm{Fe}^{3+}$ ions by the phenolic oxygen atoms of catechol. ${ }^{28}$ The intensity of the $593 \mathrm{~cm}^{-1}$ peak increases relatively compared with the other two peaks upon increasing $\mathrm{pH}$ from 3.5 to 7.4, suggesting the increase of the bidentate complex with elevating $\mathrm{pH}$ positively, which correlates with the transition from bis- to tris-coordinated $\mathrm{Fe}^{\mathrm{III}}$ species.

2.2. Mechanical Properties of PTFe Hydrogels. In the $\mathrm{PDDA} / \mathrm{TA} / \mathrm{Fe}^{\mathrm{III}}$ system, the gelation of mixtures determined by the cross-link density is mainly contributed by two parts: the connection via the electrostatic force between TA and PDDA and that via metal-ligand between $\mathrm{TA}$ and $\mathrm{Fe}^{3+} \cdot{ }^{22}$ These connections rely on the amount of pyrogallol/catechol groups, namely, the concentration of TA and $\mathrm{pH}$ values. For PTFe-1, the TA/PDDA ratio is relatively high. Therefore, a small increase of the $\mathrm{pH}$ value will lead to the formation of an efficient cross-link for gelation. At $\mathrm{pH} 0.87$, the initial mixture was a highly viscous solution containing a large amount of mono complexes $\left(G^{\prime \prime}>G^{\prime}\right)$. Further increasing the $\mathrm{pH}$ leads to a change from mono to bis complex; as a consequence, the mixture is cross-linked into a stable hydrogel $\left(G^{\prime}>G^{\prime \prime}\right)$ (Figure 3a). As for PTFe-2 to PTFe-5 hydrogels, the cross-linker density decreases with the decrease of the TA/PDDA ratio; as a consequence, the mixtures require higher $\mathrm{pH}$ values for gelation. We defined the critical gelation $\mathrm{pH}$ based on the rheological measurements (Figure $3 \mathrm{~b}$ ). Figure S2 presents the loss tangent $\left(\tan \delta=G^{\prime \prime} / G^{\prime}\right)$ of the initial PTFe solution and corresponding hydrogels at critical gelation $\mathrm{pH}$ values. As for the initial solution, the $\tan \delta$ is larger than 1 , indicating that the mixture is liquid. At the critical gelation $\mathrm{pH}$, hydrogels formed with $\tan \delta<1$. PDDA/TA bicomponent mixtures can also be gelled under a neutral $\mathrm{pH}$ condition, ${ }^{22}$ in which the critical 

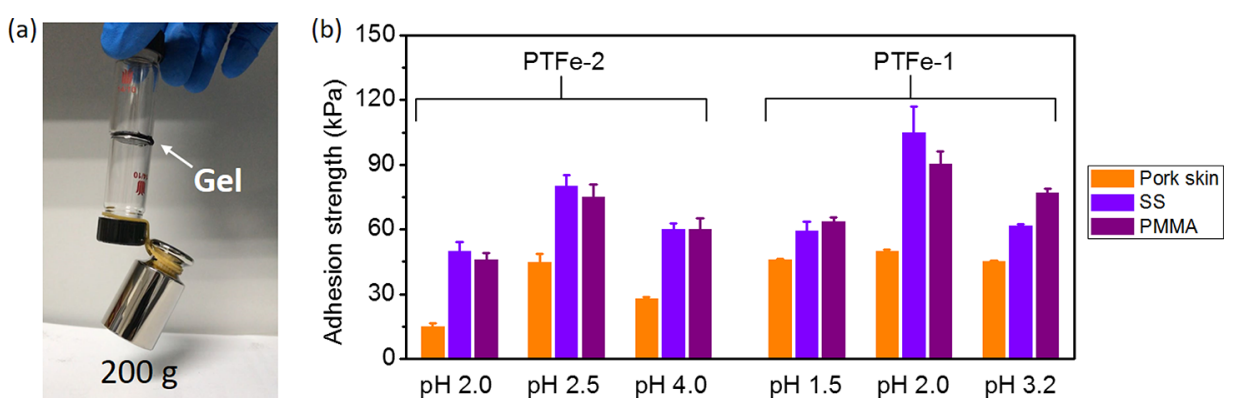

Figure 4. (a) Photograph of the test for the adhesiveness of the PTFe hydrogel. Two glass vials were glued together by the PTFe-2 (pH 2.6) hydrogel, and they could hold a $200 \mathrm{~g}$ weight. (b) Adhesive strength plotted as a function of PTFe hydrogels with different $\mathrm{pH}$ values, the tested substrates were pork skin tissue, SS, and PMMA substrates.

(a)

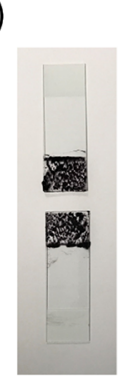
cohesive failure
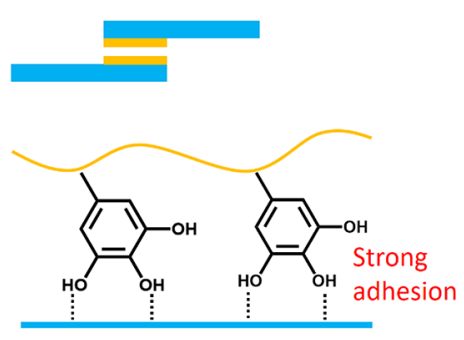

(c) Hollow-cut paper rinsed with $\mathrm{NaOH}$

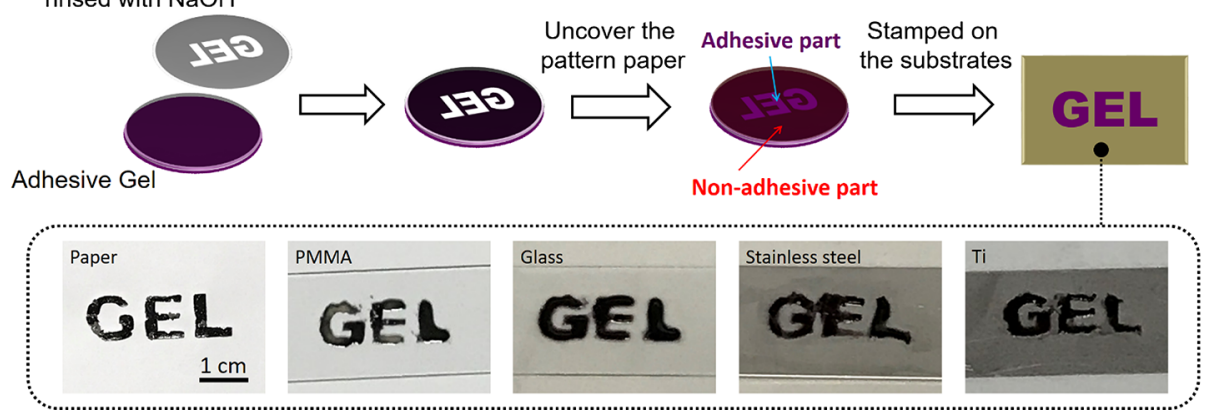

(b)

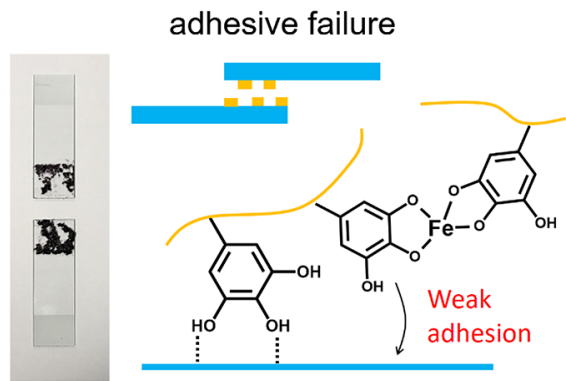

Figure 5. (a,b) Photographs of detached glass substrates and an illustration of the proposed mechanism for PTFe hydrogel adhesion at the solid interface. The hydrogels used in photographs were PTFe- $2 \mathrm{pH} 2.1$ for (a) and PTFe-2 pH 5.0 for (b). (c) Scheme of fabrication of the PTFe hydrogel with the gel pattern. The prepared hydrogel can stamp to various substrates, including paper, PMMA, glass, stainless steel, and titanium. The tested hydrogel was PTFe-2 gel, $\mathrm{pH} 2.7$.

gelation $\mathrm{pH}$ is dependent on the TA/PDDA ratio as well (Figure S3). However, compared with the tricomponent system, the gelation of the bicomponent mixture requires higher $\mathrm{pH}$ values, which may reduce the adhesiveness of hydrogels, and their mechanical strengths are much weaker than those of three-component hydrogels, resulting in weak cohesion (Figure S4). These results indicate that introducing coordinate bonds into the PDDA/TA bicomponent system is critical to generate highly adhesive hydrogels.

After gelation, the cross-link density further influenced the modulus of hydrogels, which can be tuned by changing the $\mathrm{pH}$ and the TA concentration. Angular frequency sweep measurements were conducted on all PTFe hydrogels, and $G^{\prime}$ and $G^{\prime \prime}$ values were found to be independent of the angular frequency (Figure S5), which reflects the predominant elasticity of these hydrogels. Figure $3 c$ shows the storage modulus $\left(G^{\prime}\right)$ of PTFe hydrogels with different $\mathrm{pH}$ values and different TA/PDDA weight ratios. For samples with the same TA/PDDA ratio, in $\mathrm{pH} 2-5, G^{\prime}$ highly depends on the $\mathrm{pH}$; higher $\mathrm{pH}$ corresponds to higher $G^{\prime}$. When the $\mathrm{pH}$ is above $5, G^{\prime}$ remains constant because of the saturation of coordination. For samples with different TA/PDDA weight ratios at similar $\mathrm{pH}$ values, a higher TA concentration leads to a higher degree of polymer crosslinking and higher $G^{\prime}$ (Figure 3c).

It is worth noting that PTFe hydrogels have high storage modulus under strong acidic conditions, which is never reported before. For example, the storage modulus of PTFe-1 at $\mathrm{pH} 3$ was as high as $0.15 \mathrm{MPa}$. Figure $3 \mathrm{~d}$ presents the $G^{\prime}$ values of various catechol-based hydrogels under different $\mathrm{pH}$ conditions in this work and those reported in the literature. ${ }^{9,29-36}$ Guo et al. reported catechol/chitosan hydrogels with highly substituted catechol units $(70 \%)$, the $G^{\prime}$ value of the hydrogel was only around $200 \mathrm{~Pa}$ at $\mathrm{pH} 33^{30} \mathrm{Xu}$ et al. employed the thiourea/quinone coupling for the polymer cross-linking to mimic mfps. ${ }^{31}$ In their systems, the hydrogel formed in $\mathrm{pH} 2$ has the storage modulus around $400 \mathrm{~Pa}$. The high toughness of acidic PTFe hydrogels in our case may be contributed by structural features of both TA and PDDA: (i) TA possesses a dendritic structure, which can interact with polymers through multiple sites. Such a cross-linker with high 

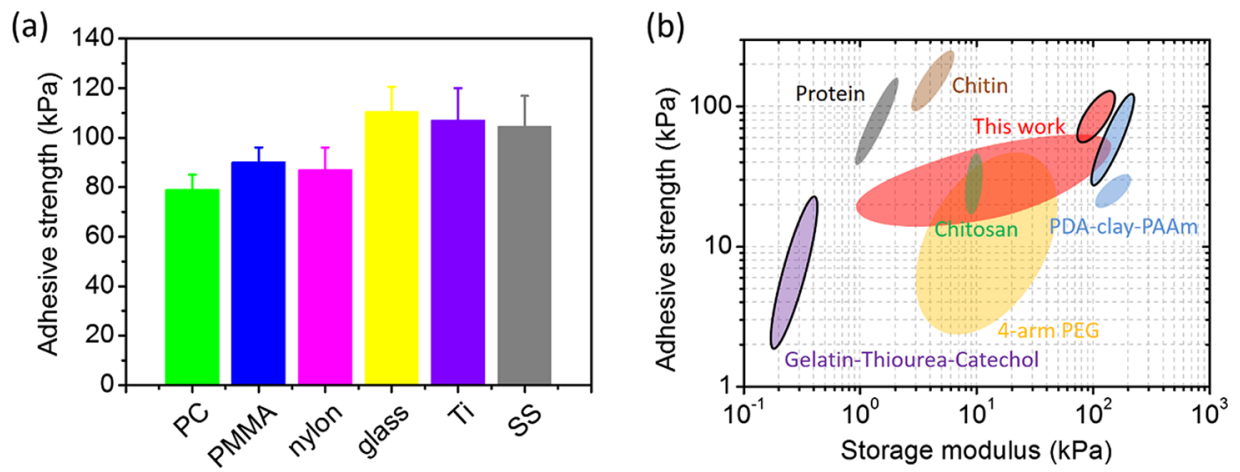

Figure 6. (a) Adhesive strength of the PTFe-1 hydrogel ( $\mathrm{pH}$ 2) to polycarbonate (PC), PMMA, nylon, glass, titanium (Ti), and SS. (b) The comparison of the adhesive strength and storage modulus of the PTFe gel in this report and various catechol-based hydrogels reported in the literature. Hydrogels include the PTFe gel prepared in this work marked as red, catechol-based chitosan hydrogels as green, ${ }^{40}$ catechol-based chitin hydrogels as brown, ${ }^{39}$ gelatin/thiourea/catechol hydrogels as purple, ${ }^{31}$ catechol-based 4-arm PEG hydrogels as yellow, ${ }^{38}$ catechol-based protein hydrogels as gray, ${ }^{34}$ and PDA-clay-PAAm hydrogels as blue. ${ }^{41}$ The graphics without outlines are attributed to the adhesiveness on tissue materials, whereas those with outlines are attributed to engineered solid materials.

functionality provides more efficient gelation than that of the single catechol group; (ii) the $\mathrm{p} K_{\mathrm{a}}$ value of TA is 8.5 , which is lower than that of the single catechol group (9.5) so that TA has a stronger acidity than catechol; ${ }^{11,21}$ (iii) PDDA is a fully charged polyelectrolyte, its polymer chains are much more rigid than a neutral polymer, such as PEG. Therefore, a high crosslinker density and a rigid polymer network guarantee the strong mechanical strength of PTFe hydrogels under strong acidic conditions. Compared with traditional catechol hydrogels prepared under basic conditions, the acidic hydrogel has advantages of preventing the oxidation of catechol units and minimizing the hydrolysis of transition metal ions, thus providing a foundation for high adhesion to engineered substrates.

2.3. Adhesiveness of PTFe Hydrogels. We further measured the adhesiveness of these PTFe hydrogels. As shown in Figure 4a, two glass vials were glued together by a thin layer of PTFe-2 ( $\mathrm{pH}$ 2.6) hydrogel, and they can be connected together by loading a $200 \mathrm{~g}$ weight. To better understand the adhesive conditions of the hydrogel, we have tested the adhesive strength of PTFe hydrogels composed of different TA/PDDA weight ratios at various $\mathrm{pH}$ values (Figure $4 \mathrm{~b})$. For the hydrogel with a different TA/PDDA ratio (PTFe-1 vs PTFe-2), the bulk adhesive strength is proportional to the TA/PDDA ratio. For samples with the same TA/PDDA ratio, with the increase of $\mathrm{pH}$ values, the bulk adhesive strength rises first and then drops. The highest adhesive strengths of each hydrogel for the pork skin, stainless steel (SS), and polymethyl methacrylate (PMMA) are 44.8 \pm 4.0, 82.1 \pm 4.7, and $75.6 \pm$ $4.0 \mathrm{kPa}$ (PTFe-2, $\mathrm{pH} 2.5) ; 49.6 \pm 0.4,104.6 \pm 12.4$, and $90.1 \pm$

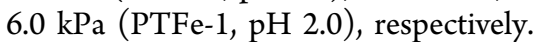

In the hydrogel, the bulk adhesive strength is determined by the gel-substrate surface interaction (surface adhesion) and the mechanical strength of gels (cohesion). In the adhesion of hydrogel-solid, delamination occurs within a gel film, which is called cohesive failure, whereas delamination occurring at the gel-solid interface is called adhesive failure. ${ }^{37}$ In our system, the strength of surface adhesion depends on the amount of free pyrogallol/catechol moieties, whereas cohesion depends on the degree of $\mathrm{TA} / \mathrm{Fe}^{\mathrm{III}}$ coordination. For samples with the same component ratio, elevating $\mathrm{pH}$ values causes the increase of the $\mathrm{TA} / \mathrm{Fe}^{\mathrm{III}}$ association degree and, in turn, the reduction of free pyrogallol/catechol groups; as a result, cohesion of hydrogels is enhanced accompanied by the decrease of surface adhesion. The balance of cohesion and adhesion endows hydrogels with the highest adhesive strength, and it is achieved at the middle $\mathrm{pH}$ range. Figure 5a,b shows photographs of detached glass substrates which were stuck by the PTFe-2 hydrogel under different $\mathrm{pH}$ conditions. In the case of hydrogel at $\mathrm{pH} 2.1$, the detachment happened at the gel body instead of the gel-solid interface (cohesive failure), indicating strong surface adhesion of pyrogallol/catechol to glass. For the hydrogel at $\mathrm{pH}$ 5.0, the detachment happened at the gel/glass interface (adhesive failure), showing weak surface adhesion of pyrogallol/catechol to glass. On the basis of facile adjustable adhesion of PTFe hydrogels, we can use the gel with different adhesiveness as a patterned stamper. As shown in Figure 5c, a hollowed-out paper with the "gel" pattern was first rinsed by $0.2 \mathrm{M} \mathrm{NaOH}$ and then covered on an adhesive PTFe hydrogel ( $\mathrm{pH} 2.7)$. The contact with the basic paper makes the adhesive hydrogel nonadhesive. After uncovering the hollowed-out paper, the hydrogel can be used to stamp various substrates with the gel pattern. $\mathrm{pH}$-manipulated adhesiveness provides a new strategy for the fabrication of patterned adhesive hydrogels. It should be mentioned that the electrostatic interaction between PDDA and a negatively charged surface is the fundamental for the adhesive property of the PDDA/TA gel. On the basis of this, TA plays a key role in the adhesion of hydrogels, through elevating hydrogel cohesion and providing additional adhesiveness for hydrogels. By fixing the concentration of PDDA, we have focused only on the influence of the TA content and the $\mathrm{pH}$ to the adhesion of hydrogels in this paper.

The PTFe hydrogel has a broad application as glue for versatile materials. We further tested the adhesive strength of the PTFe-1 ( $\mathrm{pH} 2$ ) hydrogel to glass, metals, and plastic (Figure 6a). The hydrogel can stick on these solid surfaces tightly with adhesive strengths between 80 and $120 \mathrm{kPa}$. Figure $6 \mathrm{~b}$ compares the adhesive strength of catechol-based hydrogels reported in the literature and PTFe hydrogels in this work. For tissue substrates (graphics without outlines), the majority of reported studies have utilized the oxidation of catechol to achieve strong adhesion, and the adhesive strength of these hydrogels varies considerably in different systems. ${ }^{34,38,39}$ The adhesive studies of catechol-based hydrogels to engineered materials are limited (graphics with outlines). The thioureafunctionalized acidic catechol hydrogels reported by $\mathrm{Xu}$ et al. 
(a)

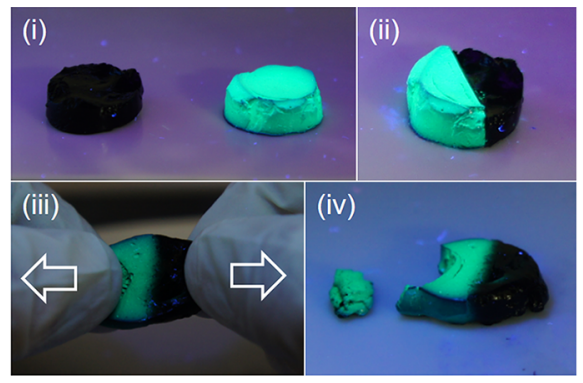

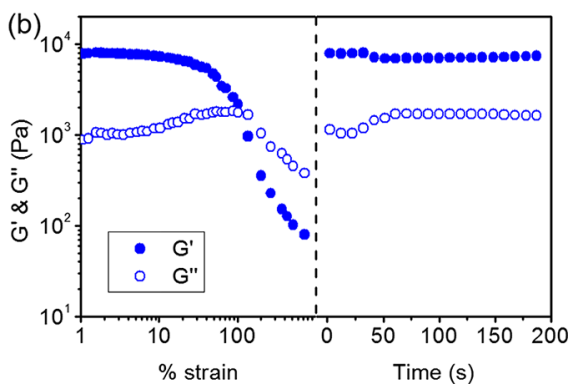

Figure 7. (a) Photographs showing the self-healing ability of the PTFe-2 ( $\mathrm{pH} 3.3)$ hydrogel. The bright-green hydrogel was doped with pyranine, and the photographs were taken under UV light (wavelength $365 \mathrm{~nm}$ ). (b) Quantitative tests of the self-healing properties of the PTFe-2 hydrogel ( $\mathrm{pH}$ 3.3). Strain was increased from 1 to $1000 \%$ at $1 \mathrm{~Hz}$, and the recovery was monitored at $1 \%$ strain with $1 \mathrm{~Hz}$.

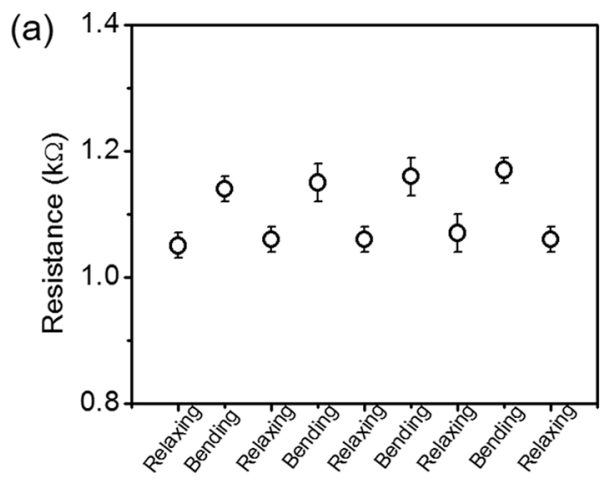

(c)

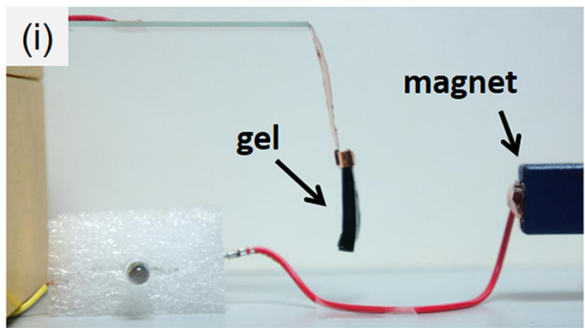

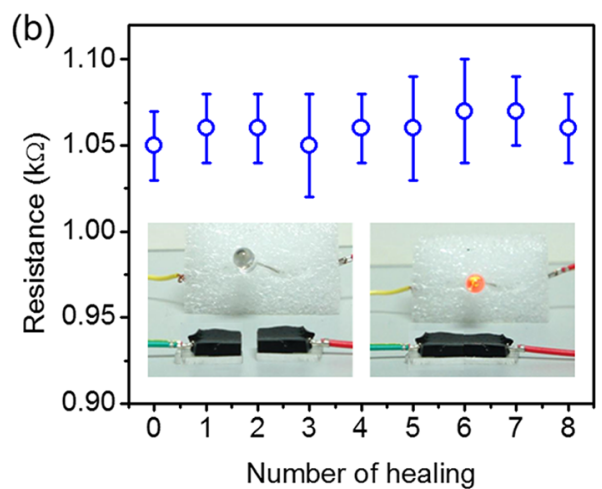

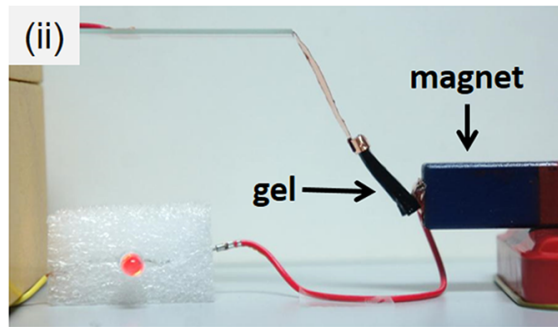

Figure 8. (a) The resistance values of the hydrogel under bending and relaxing states; the bending radius was 5 mm. (b) Resistance of the hydrogel changes as a function of repeated healing cycles. The inset photographs showed that the hydrogel conductor can self-heal in the circuit. The tested hydrogel in (a,b) was PTFe-2 ( $\mathrm{pH} 3.3)$. (c) PDDA/TA/ $\mathrm{Fe}_{3} \mathrm{O}_{4}$ magnetic gel as a power switch in the circuit.

can stick on glass with a strength of $21 \mathrm{kPa}^{31}$ Obviously, compared with catechol-based hydrogels, polyphenol TA-based hydrogels with adhesive strengths of $80-120 \mathrm{kPa}$ in this study exhibit outstanding adhesiveness, especially in engineered substrates. The dendritic structure of polyphenol TA as a cross-linker provides not only acidic condition to keep strong adhesion but also multiple binding sites with polymer chains for strong cohesion, giving rise to outstanding adhesiveness.

2.4. Self-Healing Ability of the PTFe Hydrogel. The dynamic bonds between PDDA and TA and TA and Fe $\mathrm{Fe}^{\mathrm{III}}$ endow the PTFe hydrogel with a great self-healing property. Figure $7 \mathrm{a}$ shows the self-healing test of the PTFe-2 ( $\mathrm{pH} 3.3$ ) hydrogel. Two cylinder PTFe-2 gels were cut in half (one dyed by pyranine) and put together. After cured for $1 \mathrm{~h}$, the damaged interface completely healed. Figures $7 \mathrm{~b}$ and S6 present the quantitative tests of self-healing properties of PTFe hydrogels by using rheometry. Hydrogels were subjected to increasing strains until failure, and the recovery of mechanical properties was monitored as a function of time. Upon the application of a large amplitude oscillatory strain, both $G^{\prime}$ and $G^{\prime \prime}$ values decreased and $G^{\prime}$ became lower than $G^{\prime \prime}$, indicating a network destruction. $9,35,42,43$ When the applied strain was returned to
$1 \%$, both $G^{\prime}$ and $G^{\prime \prime}$ recovered to their initial values within seconds, showing the rapid self-healing ability of PTFe hydrogels.

2.5. Ionic Conductivity of the PTFe Hydrogel. In the PDDA-based hydrogel system, a good ionic conductivity of the PDDA aqueous solution remained after the solution was crosslinked into the hydrogel. The conductivity is around $4.3 \mathrm{~S} \mathrm{~m}^{-1}$ for all tested PTFe hydrogels (Table S2). The ionic conductivity is attributed to the high concentration of the electrolyte. The resistance of PTFe hydrogels during selfhealing and relaxing-bending cycles was measured by using a multimeter. We coated the hydrogel on the PDMS substrate for a convenient operation. Figure $8 \mathrm{a}$ shows the resistance of the PTFe-2 ( $\mathrm{pH}$ 3.3) hydrogel during the bending tests. In the bending state, the resistance of the hydrogel is slightly higher than that in the relaxing state, which is caused by the deformation of the hydrogel during bending. Figure $8 \mathrm{~b}$ presents the resistance of the PTFe-2 ( $\mathrm{pH} 3.3$ ) hydrogel during the selfhealing tests. The conductivity of hydrogels after eight cut-heal cycles still recovered completely. In particular, $\mathrm{Fe}_{3} \mathrm{O}_{4}$ solid nanoparticles can replace $\mathrm{Fe}(\mathrm{III})$ salt in above hydrogel systems, resulting in magnetic, self-healable, and conductive 
hydrogels. We have tested the PDDA/TA/ $\mathrm{Fe}_{3} \mathrm{O}_{4}$ hydrogel as magnetic-responsive power switch in a circuit with an LED light. The circuit is composed of an LED bulb as the electrical load, a hydrogel as part of a conductor, and two dry batteries (2 $\times 1.5 \mathrm{~V}$ ) as the power source, as shown in Figure $8 \mathrm{c}$. When the magnet approaches, the hydrogel is attracted to the magnet, resulting in a closed circuit and lighting up of the bulb. This result indicates that polyphenol chemistry is not limited to homogeneous systems and that it may have broader application in heterogeneous systems.

\section{CONCLUSIONS}

The PDDA/TA/Fe ${ }^{\mathrm{III}}$ (PTFe) hydrogel with robust, adhesive, rapid self-healing, and ionic conductive abilities has been reported. The construction of this multifunctional hydrogel utilizes polyphenol (TA) as a supramolecular cross-linker. Compared with single catechol moieties that are covalently grafted on polymer chains, TA brings a high density of pyrogallol/catechol groups for polymers via a noncovalent pathway. As a result, the system can be cross-linked into the hydrogel in an acidic condition ( $\mathrm{pH} \mathrm{1-4).} \mathrm{The} \mathrm{high} \mathrm{cross-link}$ density with multiple functionality guarantees the strong cohesion of the hydrogel. The storage modulus of hydrogels enhanced with the increase of the TA/PDDA weight ratio, as well as the change of the $\mathrm{TA} / \mathrm{Fe}^{3+}$ coordination number (mono- to tris-complexation), and the highest storage modulus can reach to ca. $0.15 \mathrm{MPa}$ (PTFe-1 $\mathrm{pH} 3)$. On the other hand, because of the strong acidic $\mathrm{pH}$ condition preventing the oxidation of pyrogallol/catechol, a high content of TA endows the hydrogel with strong adhesion to diverse substrates, including tissues, glass, metals, and plastic. The adhesive strength of the hydrogel increased with the increase of the TA/ PDDA weight ratio at an optimized $\mathrm{pH}$ value. Moreover, PTFe hydrogels can be patterned with different adhesion because of the adjustable adhesiveness via changing the $\mathrm{pH}$. In addition, PTFe hydrogels have rapid self-healing and high ionic conductivity $\left(\sim 4.3 \mathrm{~S} \mathrm{~m}^{-1}\right)$ because of the dynamic cross-links of hydrogels and the contribution of the polyelectrolyte, respectively. Compared with traditional catechol-based hydrogels, the TA-based hydrogels can be fabricated in large scale because of low-cost materials and a simple fabrication process, which greatly benefit their further applications.

\section{EXPERIMENTAL SECTION}

4.1. Materials. PDDA solution (average $M_{\mathrm{w}} 400000-500$ 000,20 wt $\%$ in $\mathrm{H}_{2} \mathrm{O}$ ) and TA were purchased from SigmaAldrich Inc. Anhydrous ferric chloride $\left(\mathrm{FeCl}_{3}\right)$ was purchased from Aladdin Company (Shanghai, China). $\mathrm{Fe}_{3} \mathrm{O}_{4}$ nanoparticles (NPs) $(99.9 \%, 20 \mathrm{~nm}$ in diameter) were purchased from Beijing DK Nano Technology Co. LTD. Sodium hydroxide $(\mathrm{NaOH})$ and ethanol were purchased from Sinopharm Chemical Reagent Co. Ltd. All these reagents are used as received. All aqueous solutions were prepared using Millipore water.

4.2. PDDA/TA (PT) Hydrogel Formation. The hydrogels were formed by dissolving PDDA and TA in deionized water. The PDDA concentration was $150 \mathrm{mg} \mathrm{mL}^{-1}$, and the weight ratios of PDDA and TA in hydrogel samples are presented in Table S1. The viscous solution was spontaneously cross-linked into the hydrogel with increasing $\mathrm{pH}$ to the desired value by the addition of $\mathrm{NaOH}$ aqueous solution $(3 \mathrm{M})$.
4.3. PDDA/TA/Fe ${ }^{\text {III }}$ (PTFe) Hydrogel Formation. The hydrogels were formed by dissolving PDDA, TA, and $\mathrm{FeCl}_{3}$ in deionized water. The PDDA concentration was $150 \mathrm{mg} \mathrm{mL}^{-1}$, the molar ratio of TA to $\mathrm{Fe}^{3+}$ was fixed at 3:5, and the weight ratios of PDDA and TA in hydrogel samples are presented in Table 1. A high-viscosity solution was spontaneously cross-

Table 1. Weight Ratios of Various Components and the Initial pH Values in the Fabrication of Hydrogel Samples

\begin{tabular}{lll}
\multicolumn{1}{c}{ sample name } & weight ratio of PDDA/TA/ $\mathrm{FeCl}_{3}{ }^{a}$ & \multicolumn{1}{c}{$\begin{array}{c}\text { initial solution } \\
\mathrm{pH}\end{array}$} \\
PTFe-1 & $2: 1: 0.16$ & 0.87 \\
PTFe-2 & $4: 1: 0.16$ & 1.3 \\
PTFe-3 & $10: 1: 0.16$ & 1.5 \\
PTFe-4 & $15: 1: 0.16$ & 1.7 \\
PTFe-5 & $20: 1: 0.16$ & 2.1 \\
sample name & weight ratio of PDDA/TA/ $/ \mathrm{Fe}_{3} \mathrm{O}_{4}$ & initial solution \\
& $\mathrm{NPs}$ & $\mathrm{pH}$ \\
PDDA/TA/Fe & 2.9 \\
${ }_{\text {The molar ratio of }}$ TA to $\mathrm{Fe}^{3+}$ was fixed at 3:5. & \\
& $4: 1: 2$ & \\
\end{tabular}

linked into the hydrogel with increasing $\mathrm{pH}$ to the desired value by the addition of $\mathrm{NaOH}$ aqueous solution $(3 \mathrm{M})$. On the basis of our previous study, the PDDA/TA precipitate can be formed when the TA/PDDA weight ratio is up to 0.6 in a wide $\mathrm{pH}$ condition $(\mathrm{pH} 1-11) .^{22}$ Below this value, there is no precipitate in TA/PDDA mixtures. In this study, the highest TA/PDDA weight ratio is maintained at 0.5 for avoiding the precipitation of TA/PDDA in all experiments ( $\mathrm{pH} 1-11)$. It should be mentioned that homogenous PTFe-1 and PTFe- 2 gels were obtained only under strong acidic conditions; the gels became too rigid to form homogenous materials under weak acidic conditions.

4.4. PDDA/TA/ $\mathrm{Fe}_{3} \mathrm{O}_{4}$ Hydrogel Formation. $\mathrm{Fe}_{3} \mathrm{O}_{4} \mathrm{NPs}$ were first dispersed in the TA aqueous solution by sonication and stirring under an ice-water bath (sonication for $20 \mathrm{~s}$ with 10 s break, 180 cycles, the sonication power was $150 \mathrm{~W}$ ). Then the suspension was gradually mixed with the PDDA solution under sonication and stirring under the same condition as mentioned above. The final PDDA concentration was $150 \mathrm{mg} \mathrm{mL}^{-1}$. The weight ratios of PDDA, TA, and $\mathrm{Fe}_{3} \mathrm{O}_{4}$ NPs are presented in Table 1. A high-viscosity solution was spontaneously crosslinked into the hydrogel with increasing $\mathrm{pH}$ to the desired value by the addition of $\mathrm{NaOH}$ aqueous solution ( $3 \mathrm{M}$ ).

4.5. Characterizations. The $\mathrm{pH}$ of hydrogels was measured using the Mettler-Toledo LE427 $\mathrm{pH}$ puncture electrode. The electrical conductivity was measured by a Mettler-Toledo conductivity meter equipped with an InLab 731 electrode. The flexible and self-healing properties during the conduction of the hydrogel were measured by using a multimeter. Raman spectra were measured by using Raman spectroscopy (HORIBA Scientific XploRA PLUS) with a 785 $\mathrm{nm}$ laser light as the excitation source, and the laser power was $25 \mathrm{~mW}$. The absorbance of the hydrogel was measured by holding the gel between two quartz cover slips and placing them directly in the light path of the spectrophotometer (Varian Cary 50). Relative fractions of mono-, bis-, and triscatechol $/ \mathrm{Fe}^{3+}$ complexes were deduced by fitting a multi-Gauss function in an iterative manner to the collected absorption profiles. ${ }^{9}$ The characteristic mono-, bis- and tris-complex peaks were located at 615,574 , and $520 \mathrm{~nm}$, respectively. The areas of these characteristic peaks were converted into the relative 
fractions by normalizing them to the maximum area value for each characteristic peak.

The rheological properties of hydrogels were tested using a rheometer (Thermo Scientific HAAKE) with a parallel-plate geometry ( $35 \mathrm{~mm}$ diameter rotating top plate). The mechanical properties were measured by performing angular frequency sweeps in the linear viscoelastic range at a strain of 5\%, while monitoring the storage modulus $\left(G^{\prime}\right)$ and loss modulus $\left(G^{\prime \prime}\right)$. The self-healing properties were investigated by straining the gels from 1 to $1000 \%$ strain at a frequency of $1 \mathrm{~Hz}$. Then, the recovery of the hydrogel was measured by monitoring the storage modulus at $1 \mathrm{~Hz}$ and $1 \%$ strain. All characterizations were performed at $25^{\circ} \mathrm{C}$, and each sample was tested three times in parallel.

Tensile-adhesion measurements of PTFe hydrogels were performed by a universal testing machine [(UTM), INSTRON 5583]. The substrates such as PC, PMMA, nylon, glass, $\mathrm{Ti}$, stainless steel (SS), and pork skin tissue were commercially available. The engineered solid specimens $(25 \mathrm{~mm} \times 100 \mathrm{~mm}$ $\times 1 \mathrm{~mm}$ ) were washed with deionized water and ethanol and then dried before use. The pork skin tissues were cut into 25 $\mathrm{mm} \times 25 \mathrm{~mm}$ squares and attached to aluminum fixtures by using cyanoacrylate glue before the test. ${ }^{34}$ To adhere to the substrate, a hydrogel with $20 \mathrm{~mm} \times 20 \mathrm{~mm} \times 1 \mathrm{~mm}$ was placed between two specimens and compressed with a $100 \mathrm{~g}$ weight for $2 \mathrm{~min}$. The adhered plates were clamped to the UTM and then separated at a crosshead speed of $10 \mathrm{~mm} \mathrm{~s}^{-1}$. The adhesion strength was calculated by the measured maximum load divided by the bonded area. Each sample was tested five times in parallel.

\section{ASSOCIATED CONTENT}

\section{S Supporting Information}

The Supporting Information is available free of charge on the ACS Publications website at DOI: 10.1021/acsomega.7b01067. UV-vis absorption PDDA/TA/Fe ${ }^{\mathrm{III}}$ complex at $\mathrm{pH}$ values; loss tangent plotted as a function of the angular frequency of the initial PTFe solution and that of hydrogels at critical gelation $\mathrm{pH}$ values; critical gelation $\mathrm{pH}$ of PT hydrogels; storage $G^{\prime}$ and loss $G^{\prime \prime}$ moduli of PTFe hydrogels with different angular frequency sweeps; quantitative tests of the self-healing properties of PTFe hydrogels; and the weight ratios of various components and the initial $\mathrm{pH}$ values in the fabrication of PDDA/TA bicomponent hydrogels (PDF)

\section{AUTHOR INFORMATION}

\section{Corresponding Author}

*E-mail: jinzx@ruc.edu.cn (Z.J.).

\section{ORCID}

Zhaoxia Jin: 0000-0002-6108-0636

\section{Notes}

The authors declare no competing financial interest.

\section{ACKNOWLEDGMENTS}

The authors gratefully acknowledge the National Natural Science Foundation of China (grants 21374132 and 51673210) for financial support.

\section{REFERENCES}

(1) Lee, B. P.; Messersmith, P. B.; Israelachvili, J. N.; Waite, J. H. Mussel-Inspired Adhesives and Coatings. Annu. Rev. Mater. Res. 2011, 41, 99-132.

(2) Lin, Q.; Gourdon, D.; Sun, C.; Holten-Andersen, N.; Anderson, T. H.; Waite, J. H.; Israelachvili, J. N. Adhesion Mechanisms of the Mussel Foot Proteins mfp-1 and mfp-3. Proc. Natl. Acad. Sci. U.S.A. 2007, 104, 3782-3786.

(3) Forooshani, P. K.; Lee, B. P. Recent Approaches in Designing Bioadhesive Materials Inspired by Mussel Adhesive Protein. J. Polym. Sci., Part A: Polym. Chem. 2017, 55, 9-33.

(4) Harrington, M. J.; Masic, A.; Holten-Andersen, N.; Waite, J. H.; Fratzl, P. Iron-Clad Fibers: A Metal-Based Biological Strategy for Hard Flexible Coatings. Science 2010, 328, 216-220.

(5) Nicklisch, S. C. T.; Waite, J. H. Mini-review: The Role of Redox in Dopa-Mediated Marine Adhesion. Biofouling 2012, 28, 865-877.

(6) Hwang, D. S.; Zeng, H.; Masic, A.; Harrington, M. J.; Israelachvili, J. N.; Waite, J. H. Protein- and Metal-dependent Interactions of a Prominent Protein in Mussel Adhesive Plaques. J. Biol. Chem. 2010, 285, 25850-25858.

(7) Yu, J.; Wei, W.; Danner, E.; Ashley, R. K.; Israelachvili, J. N.; Waite, J. H. Mussel Protein Adhesion Depends on Interprotein ThiolMediated Redox Modulation. Nat. Chem. Biol. 2011, 7, 588-590.

(8) Yang, B.; Lim, C.; Hwang, D. S.; Cha, H. J. Switch of Surface Adhesion to Cohesion by Dopa-Fe ${ }^{3+}$ Complexation, in Response to Microenvironment at the Mussel Plaque/Substrate Interface. Chem. Mater. 2016, 28, 7982-7989.

(9) Holten-Andersen, N.; Harrington, M. J.; Birkedal, H.; Lee, B. P.; Messersmith, P. B.; Lee, K. Y. C.; Waite, J. H. pH-Induced MetalLigand Cross-Links Inspired by Mussel Yield Self-Healing Polymer Networks with Near-Covalent Elastic Moduli. Proc. Natl. Acad. Sci. U.S.A. 2011, 108, 2651-2655.

(10) Li, Y.; Lee, B. P. Biomimetic Approach to Designing Adhesive Hydrogels: From Chemistry to Application. In Carbon Nanomaterials for Biomedical Applications; Zhang, M., Naik, R. R., Dai, L., Eds.; Springer International Publishing: Cham, 2016; pp 481-500.

(11) Krogsgaard, M.; Nue, V.; Birkedal, H. Mussel-Inspired Materials: Self-Healing through Coordination Chemistry. Chem.Eur. J. 2015, 22, 844-857.

(12) Li, L.; Smitthipong, W.; Zeng, H. Mussel-Inspired Hydrogels for Biomedical and Environmental Applications. Polym. Chem. 2015, 6, $353-358$.

(13) Lee, H.; Scherer, N. F.; Messersmith, P. B. Single-Molecule Mechanics of Mussel Adhesion. Proc. Natl. Acad. Sci. U.S.A. 2006, 103, 12999-13003.

(14) Yu, J.; Wei, W.; Danner, E.; Israelachvili, J. N.; Waite, J. H. Effects of Interfacial Redox in Mussel Adhesive Protein Films on Mica. Adv. Mater. 2011, 23, 2362-2366.

(15) Anderson, T. H.; Yu, J.; Estrada, A.; Hammer, M. U.; Waite, J. H.; Israelachvili, J. N. The Contribution of DOPA to SubstratePeptide Adhesion and Internal Cohesion of Mussel-Inspired Synthetic Peptide Films. Adv. Funct. Mater. 2010, 20, 4196-4205.

(16) Yavvari, P. S.; Srivastava, A. Robust, Self-Healing Hydrogels Synthesised from Catechol Rich Polymers. J. Mater. Chem. B 2015, 3, 899-910.

(17) Zhang, Y.; Zhou, N.; Akella, S.; Kuang, Y.; Kim, D.; Schwartz, A.; Bezpalko, M.; Foxman, B. M.; Fraden, S.; Epstein, I. R.; Xu, B. Active Cross-Linkers that Lead to Active Gels. Angew. Chem., Int. Ed. 2013, 52, 11494-11498.

(18) Auriemma, M.; Piscitelli, A.; Pasquino, R.; Cerruti, P.; Malinconico, M.; Grizzuti, N. Blending Poly(3-hydroxybutyrate) with Tannic Acid: Influence of A Polyphenolic Natural Additive on the Rheological and Thermal Behavior. Eur. Polym. J. 2015, 63, 123131.

(19) Makris, D. P.; Boskou, G.; Andrikopoulos, N. K. Polyphenolic Content and In Vitro Antioxidant Characteristics of Wine Industry and Other Agri-Food Solid Waste Extracts. J. Food Compos. Anal. 2007, 20, 125-132. 
(20) Shutava, T.; Prouty, M.; Kommireddy, D.; Lvov, Y. pH Responsive Decomposable Layer-by-Layer Nanofilms and Capsules on the Basis of Tannic Acid. Macromolecules 2005, 38, 2850-2858.

(21) Erel-Unal, I.; Sukhishvili, S. A. Hydrogen-Bonded Multilayers of a Neutral Polymer and a Polyphenol. Macromolecules 2008, 41, 39623970.

(22) Fan, H. L.; Wang, L.; Feng, X.; Bu, Y.; Wu, D.; Jin, Z. Supramolecular Hydrogel Formation Based on Tannic Acid. Macromolecules 2017, 50, 666-676.

(23) Ejima, H.; Richardson, J. J.; Liang, K.; Best, J. P.; van Koeverden, M. P.; Such, G. K.; Cui, J.; Caruso, F. One-Step Assembly of Coordination Complexes for Versatile Film and Particle Engineering. Science 2013, 341, 154-157.

(24) Li, W.; Bing, W.; Huang, S.; Ren, J.; Qu, X. Mussel Byssus-Like Reversible Metal-Chelated Supramolecular Complex Used for Dynamic Cellular Surface Engineering and Imaging. Adv. Funct. Mater. 2015, 25, 3775-3784.

(25) Rahim, M. A.; Kempe, K.; Müllner, M.; Ejima, H.; Ju, Y.; van Koeverden, M. P.; Suma, T.; Braunger, J. A.; Leeming, M. G.; Abrahams, B. F.; Caruso, F. Surface-Confined Amorphous Films from Metal-Coordinated Simple Phenolic Ligands. Chem. Mater. 2015, 27, 5825-5832.

(26) Rahim, M. A.; Björnmalm, M.; Suma, T.; Faria, M.; Ju, Y.; Kempe, K.; Müllner, M.; Ejima, H.; Stickland, A. D.; Caruso, F. Metal-Phenolic Supramolecular Gelation. Angew. Chem., Int. Ed. 2016, 55, 13803-13807.

(27) Rahim, M. A.; Björnmalm, M.; Bertleff-Zieschang, N.; Besford, Q.; Mettu, S.; Suma, T.; Faria, M.; Caruso, F. Rust-Mediated Continuous Assembly of Metal-Phenolic Networks. Adv. Mater. 2017, 29, 1606717.

(28) Taylor, S. W.; Chase, D. B.; Emptage, M. H.; Nelson, M. J.; Waite, J. H. Ferric Ion Complexes of a DOPA-Containing Adhesive Protein from Mytilus edulis. Inorg. Chem. 1996, 35, 7572-7577.

(29) Ghadban, A.; Ahmed, A. S.; Ping, Y.; Ramos, R.; Arfin, N.; Cantaert, B.; Ramanujan, R. V.; Miserez, A. Bioinspired pH and Magnetic Responsive Catechol-Functionalized Chitosan Hydrogels with Tunable Elastic Properties. Chem. Commun. 2016, 52, 697-700.

(30) Guo, Z.; Ni, K.; Wei, D.; Ren, Y. Fe ${ }^{3+}$-Induced Oxidation and Coordination Cross-Linking in Catechol-Chitosan Hydrogels Under Acidic pH Conditions. RSC Adv. 2015, 5, 37377-37384.

(31) Xu, Y. J.; Wei, K.; Zhao, P.; Feng, Q.; Choi, C. K. K.; Bian, L. Preserving the Adhesion of Catechol-Conjugated Hydrogels by Thiourea-Quinone Coupling. Biomater. Sci. 2016, 4, 1726-1730.

(32) Barrett, D. G.; Fullenkamp, D. E.; He, L.; Holten-Andersen, N.; Lee, K. Y. C.; Messersmith, P. B. pH-Based Regulation of Hydrogel Mechanical Properties Through Mussel-Inspired Chemistry and Processing. Adv. Funct. Mater. 2013, 23, 1111-1119.

(33) Liu, Y.; Meng, H.; Konst, S.; Sarmiento, R.; Rajachar, R.; Lee, B. P. Injectable Dopamine-Modified Poly(ethylene glycol) Nanocomposite Hydrogel with Enhanced Adhesive Property and Bioactivity. ACS Appl. Mater. Interfaces 2014, 6, 16982-16992.

(34) Kim, B. J.; Oh, D. X.; Kim, S.; Seo, J. H.; Hwang, D. S.; Masic, A.; Han, D. K.; Cha, H. J. Mussel-Mimetic Protein-Based Adhesive Hydrogel. Biomacromolecules 2014, 15, 1579-1585.

(35) Ceylan, H.; Urel, M.; Erkal, T. S.; Tekinay, A. B.; Dana, A.; Guler, M. O. Mussel Inspired Dynamic Cross-Linking of Self-Healing Peptide Nanofiber Network. Adv. Funct. Mater. 2013, 23, 2081-2090.

(36) Krogsgaard, M.; Hansen, M. R.; Birkedal, H. Metals \& Polymers in the Mix: Fine-Tuning the Mechanical Properties \& Color of SelfHealing Mussel-Inspired Hydrogels. J. Mater. Chem. B 2014, 2, 82928297.

(37) Gebbie, M. A.; Wei, W.; Schrader, A. M.; Cristiani, T. R.; Dobbs, H. A.; Idso, M.; Chmelka, B. F.; Waite, J. H.; Israelachvili, J. N. Tuning Underwater Adhesion with Cation $-\pi$ Interactions. Nat. Chem. 2017, 9, 473-479.

(38) Barrett, D. G.; Bushnell, G. G.; Messersmith, P. B. Mechanically Robust, Negative-Swelling, Mussel-Inspired Tissue Adhesives. Adv. Healthcare Mater. 2013, 2, 745-755.
(39) Oh, D. X.; Kim, S.; Lee, D.; Hwang, D. S. Tunicate-Mimetic Nanofibrous Hydrogel Adhesive with Improved Wet Adhesion. Acta Biomater. 2015, 20, 104-112.

(40) Ryu, J. H.; Lee, Y.; Do, M. J.; Jo, S. D.; Kim, J. S.; Kim, B.-S.; Im, G.-I.; Park, T. G.; Lee, H. Chitosan-g-Hematin: Enzyme-Mimicking Polymeric Catalyst for Adhesive Hydrogels. Acta Biomater. 2014, 10, 224-233.

(41) Han, L.; Lu, X.; Liu, K.; Wang, K.; Fang, L.; Weng, L.-T.; Zhang, H.; Tang, Y.; Ren, F.; Zhao, C.; Sun, G.; Liang, R.; Li, Z. MusselInspired Adhesive and Tough Hydrogel Based on Nanoclay Confined Dopamine Polymerization. ACS Nano 2017, 11, 2561-2574.

(42) Hui, Y.; Wen, Z.-B.; Pilate, F.; Xie, H.; Fan, C.-J.; Du, L.; Liu, D.; Yang, K.-K.; Wang, Y.-Z. A Facile Strategy to Fabricate HighlyStretchable Self-Healing Poly(Vinyl Alcohol) Hybrid Hydrogels Based on Metal-Ligand Interactions and Hydrogen Bonding. Polym. Chem. 2016, 7, 7269-7277.

(43) Krogsgaard, M.; Andersen, A.; Birkedal, H. Gels and Threads: Mussel-Inspired One-Pot Route to Advanced Responsive Materials. Chem. Commun. 2014, 50, 13278-13281. 